특집논문-09-14-4-02

\title{
Retrieval of Identical Clothing Images Based on Non-Static Color Histogram Analysis
}

\author{
Yoo-Joo Choi ${ }^{\text {a) }}$, Nam-Mee Moon ${ }^{\text {b) }}$, and Ku-Jin $\mathrm{Kim}^{\mathrm{c})^{\ddagger}}$
}

\begin{abstract}
In this paper, we present a non-static color histogram method to retrieve clothing images that are similar to a query clothing. Given clothing area, our method automatically extracts major colors by using the octree-based quantization approach[16]. Then, a color palette that is composed of the major colors is generated. The feature of each clothing, which can be either a query or a database clothing image, is represented as a color histogram based on its color palette. We define the match color bins between two possibly different color palettes, and unify the color palettes by merging or deleting some color bins if necessary. The similarity between two histograms is measured by using the weighted Euclidean distance between the match color bins, where the weight is derived from the frequency of each bin. We compare our method with previous histogram matching methods through experiments. Compared to HSV cumulative histogram-based approach, our method improves the retrieval precision by $13.7 \%$ with less number of color bins.
\end{abstract}

Keywords: color quantization, color identification, histogram, clothing identification

\section{Introduction}

The recent moderns have been exposed to a lot of multimedia data in the daily life. Not only image data but also various kinds of sound and movie data related to the everyday life have been dramatically increased. Therefore, the effective and interactive content-based retrieval methods

a) 서울벤처정보대학원대학교 컴퓨터응용기술학과

Dept. of Computer Science \& Application Seoul Univ. of Venture \& Information

b) 호서대학교 벤처전문대학원 IT 응용기술학과

Dept. of IT Application Hoseo Graduate School of Venture Hoseo University

c) 경북대학교 컴퓨터공학과

Dept. of Computer Engineering Kyungpook National University

\# 교신저자 : 김구진(kujinkim@knu.ac.kr)

※ This research is supported by Foundation of Ubiquitous Computing and Networking project(UCN), the Ministry of Knowledge Economy (MKE) 21st Centry Frontier R\&D Program in Korea and a result of subproject UCN (09C1-T3-12T).

- 접수일(2009년3월13일),수정일(2009년6월30일), 게재확정일(2009년7 월20일) for a large multimedia database have been actively researched in recent years ${ }^{[1-4]}$. Most of content-based retrieval systems for an image database analyze color and texture information to define the image features and to discriminate the similarity of different images. Especially, the color analysis is a basic core technology for the robust image retrieval.

Color analysis technologies have been also applied to the smart surveillance system to robustly track pedestrians from CCD camera images captured in real-time. In the multiple camera environment, the clothing colors and positions of the pedestrians for each camera are analyzed and compared with the pedestrian information of the different camera in order to discriminate the same pedestrian ${ }^{[5-6]}$. Especially, the clothing color of the pedestrian is a primary analysis factor for person identification in the multiple camera environment that doesn't include the overlapping 
FOV(Field of View) of multiple cameras.

In this paper, we propose a method to solve the clothing identification problem, where the problem is to retrieve the clothing images that are similar to a given query clothing image. To solve the clothing identification problem, we present a novel algorithm that measures the color similarity between clothing images based on the quantized color histogram. Generally, more number of color bins is required for more precise measurement of color similarity between two images. On the other hand, more number of color bins causes high computational load. Our method targets to retrieve identical clothing images with higher precision using less number of color bins compared to the previous color histogram matching approaches.

For each given image, clothing identification system automatically detects the clothing areas. Then, the system automatically extracts major colors from the clothing area based on the octree-based quantization approach ${ }^{[14]}$. The major colors compose a color palette for the clothing area. The feature of each clothing, which can be either a query clothing or a clothing image from a database, is represented as a color histogram based on its color palette. To measure the similarity between two color histograms constructed from possibly different color palettes, we define the match color bins. After finding match color bins between two color histograms, some color bins can be merged or deleted from the histogram if necessary. Then, we compute the similarity between two histograms by using the weighted Euclidean distance between the match color bins, where the weight is derived from the frequency of each bin. By using local color histograms, we also try to overcome the weakness of the traditional histogram-based approaches that contains insufficient spatial information.

The advantages of our approach are as follows.

1. Real-time processing for the clothing identification

2. Extraction of the non-distorted quantized colors for each image by applying octree-based color quantization

3. Extraction of spatial color information by using local histograms

The organization of this paper is as follows. We introduce the related work in Section 2 and present the overview of our algorithm in Section 3. We explain the method to detect the clothing area in Section 4. The algorithm to measure the color similarity between two clothing images is proposed in Section 5. The experimental results are presented in Section 6 and we conclude this paper in Section 7.

\section{Related Work}

Content-based image retrieval methods use the visual information such as color, texture and shape to define the image features and to retrieve the similar images to given query $^{[1-4,7]}$. Among the visual information, especially color is one of the most efficient tools to recognize specific objects and to define the similarity between the objects. This is due to the reason that color features are not seriously affected by the direction of objects and minute changes of the camera viewpoint.

Up to now, histograms have been widely used as an intuitive and efficient tool to represent the color information of an image. The similarity between images can be simply but effectively measured by the sum of $L_{1}$ or $L_{2}$ distances between matching bins of the histograms ${ }^{[8]}$. The histogram intersection method $^{[9]}$ also has been widely used to compute the similarity.

Nevertheless the usefulness of histograms, there is a serious limitation in using histogram-based approaches since the histograms do not include the spatial information. $\mathrm{CCV}$ (Color Coherence Vector)-based approaches ${ }^{[10]}$ have 
been researched in order to represent the spatial information with colors, but these approaches usually require the high computation complexity.

Image analysis methods with image quantization have been researched to increase the time efficiency of image retrieval. Kang et al. ${ }^{[1]}$ quantized images by 64 color bins, and Tous et al. ${ }^{[12]}$ grouped colors into eight different color categories: grey, blue, green, yellow, orange, red, pink and purple. These approaches utilize fixed color palettes, so the distorted quantized color can be extracted by transforming an original color to a totally different color in the color palette.

\section{Algorithm Overview}

The overview of our algorithm is presented in Fig. 1. Our algorithm first detects clothing areas in given images and applies the octree-based quantization to extract major colors of each clothing area. Two possibly different color palettes are constructed for two clothing areas. Based on the color palette, six non-static color histograms are generated for six subregions in each clothing area. After applying color bin matching between two non-static color histograms from two different clothing area, the similarity between two clothing areas is measured.

\section{Detection of Clothing Area}

To extract the clothing area from given image, we first detect the face area. The body area is detected from the face area at a fixed distance in lower direction and its size is decided according to the size of the face area. The clothing area is supposed to be the same as the body area.

We detect the skin blobs from the grid image using

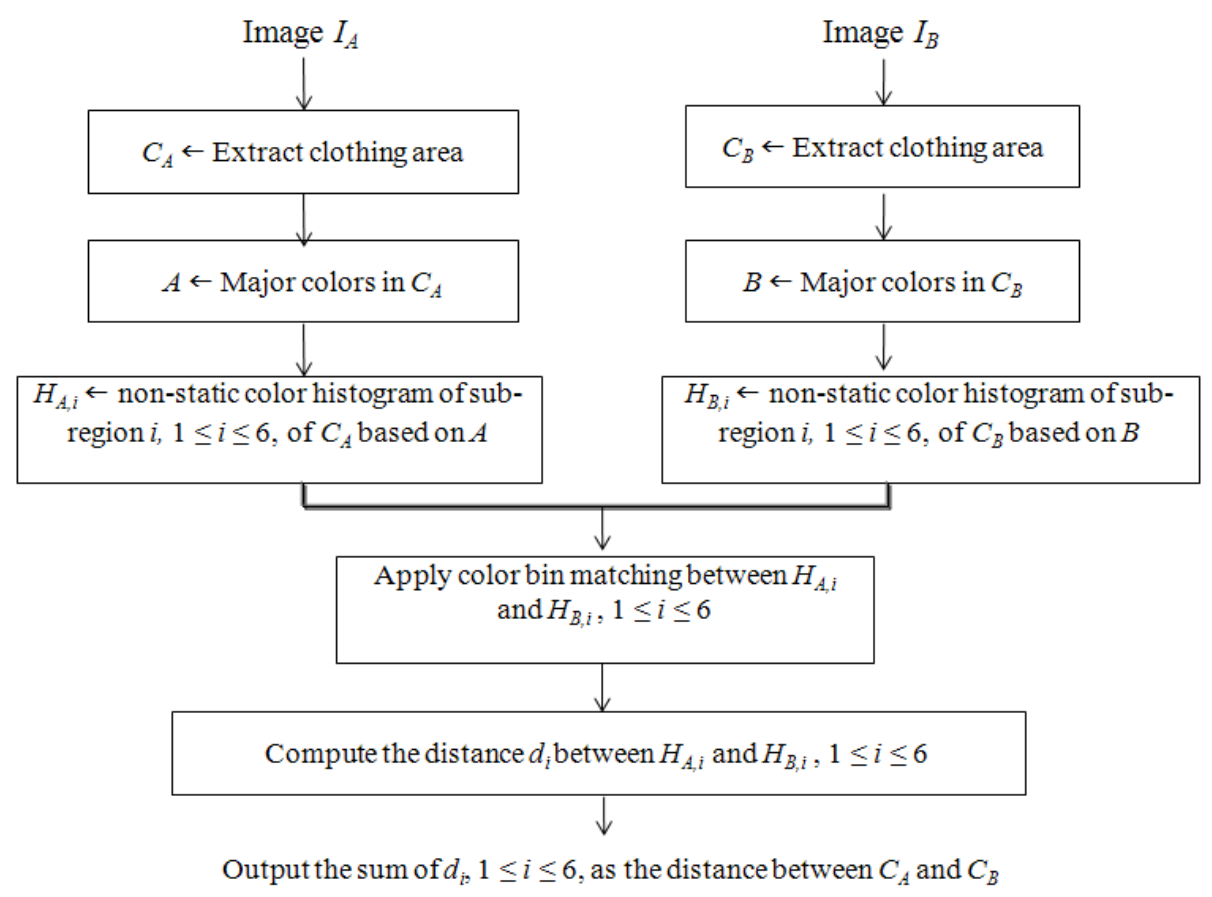

그림 1. 의상 영상 간 유사도 계산을 위한 순서도

Fig. 1. Flowchart for computing similarity between two clothing images. 
CCL(Connected-Component Labeling) algorithm ${ }^{[13]}$. Background subtraction images usually include the pixel noises or holes in the skin area. Therefore, when we perform the labeling of the skin area by directly applying CCL to the background subtraction image, unexpected labeling results can be acquired due to the noises and holes. On the other hand, we can prevent detecting too small skin blobs by using an image patch as a unit in clustering of skin pixels and increase the performance due to reduction of the resolution by using the grid image ${ }^{[14]}$.

For each skin blob, AdaBoost algorithm ${ }^{[15]}$ is applied to check whether the face features are included. AdaBoost algorithm returns 1 if the input blob includes the face feature. It returns 0 , otherwise. If the face feature is detected, the size of the face blob is adjusted to the detected face size. Then, the clothing area is detected by finding the area that is apart from the face blob at a fixed distance in lower direction.

We classify the camera types into two categories based on the height of camera locations. The height of Type A cameras is low, and that of Type B cameras is high. The viewpoints of Type A cameras are almost at the front of the pedestrian. Type B cameras are located at the higher place than the average height of male adults, possibly at the ceiling. In the case of Type A camera, the distance between the face and clothing areas is $1 / 4$ height of the face
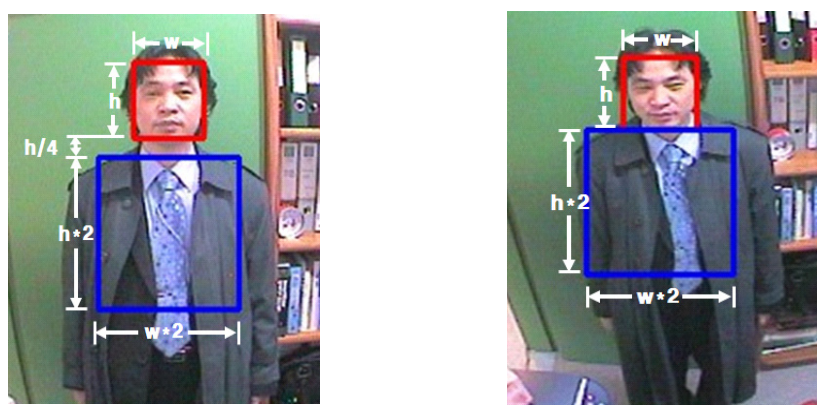

그림 2. 두 대의 카메라 영상에서 감지된 얼굴 및 의상 영역

Fig. 2. The detected face and clothing areas in two types of camera images (left: Type A, Right: Type B) blob. For Type B cameras, clothing area is stuck to the face area, so the size of clothing area is decided by scaling twice that of the face area. Fig. 2 compares the camera images of Type A and B by showing the detected clothing areas(blue rectangle) and face areas(red rectangle).

\section{Clothing Identification}

To find the similar clothing images, we measure the color similarity between them. For efficient color analysis, we quantize the colors used in clothing images. In this section, we explain the octree-based color quantization method and present an algorithm that measure the color similarity.

\section{Extraction of Major Colors Using Octree-Based Quantization}

The color quantization problem is representing a full color image by using a restricted number of colors, which is called color palettes, under the constraint that the result image looks similar to the original one. To solve the color quantization problem, construction of an optimal color palette and the optimal mapping of each pixel of the image to a color from the palette ${ }^{[17]}$. In our approach, we compose a color palette containing 10 or less major colors for each clothing area that originally represented by up to 224 colors. In constructing a color palette, an octree-based color quantization technique ${ }^{[16]}$ is used.

The simplest way to quantize colors is "uniform quantization" that divides the RGB cube into equal slices in each dimension and construct a color palette composed of the major colors from those slices. The uniform quantization algorithm is fairly fast, but suffers from the severe image degradation because they do not consider two facts: i) the major colors of the image, and ii) the number of different colors in the image. 
On the other hand, the octree-based quantization produces a color quantized image with little degradation regardless of the number of different colors in the original image. It hierarchically slices the color space to find the major colors in the image, so the result color palette can be composed of the colors mainly represented in the image. In generating color palettes, the octree-based approach takes longer time than the uniform quantization. However, octree-based approach is more appropriate in analyzing the clothing colors, since the number of colors used in a clothing area tends to be low and the area usually consists of several set of similar colors.

In most of color quantization approaches that select limited $K$ colors out of the possible colors, the image is read sequentially and the first $K$ different colors are used as initial entries to the color table. If another color is added, which means that the processed part of the image has $K+1$ different colors, near neighbors are merged into one and substituted by their mean. This step is repeated for every additional color, so that no more than $K$ major colors are left at any moment. For this process, a data structure is needed to help the quick detection of closest colors in the color space.

An octree is well suited for this usage. The RGB cube can easily be administered by an octree. When the color components of each pixel, red, green, and blue, in the image have the range from 0 to 255 , respectively, an octree of depth eight sufficiently represents all possible colors. Each color component corresponds to an axis of the 3-dimensional space, and each color value corresponds to the coordinate values.

Octree consists of a number of nodes, each of them has up to 8 children. When an index gives the position of the color in the RGB cube, the index is represented as a branch in the octree. Every exact color in the RGB cube is represented by a leaf node placed at level eight in the octree. Intermediate nodes represent subcubes of the RGB space. The node placed at a level 1 in the octree corresponds to the subcube with size $27-1 \times 27-1 \times 27-1$ in the color space. Therefore, the level of a node can be used to measure the maximum distance between major colors. Fig. 3 explains the mapping process of the RGB value $(140,200,255)$ to

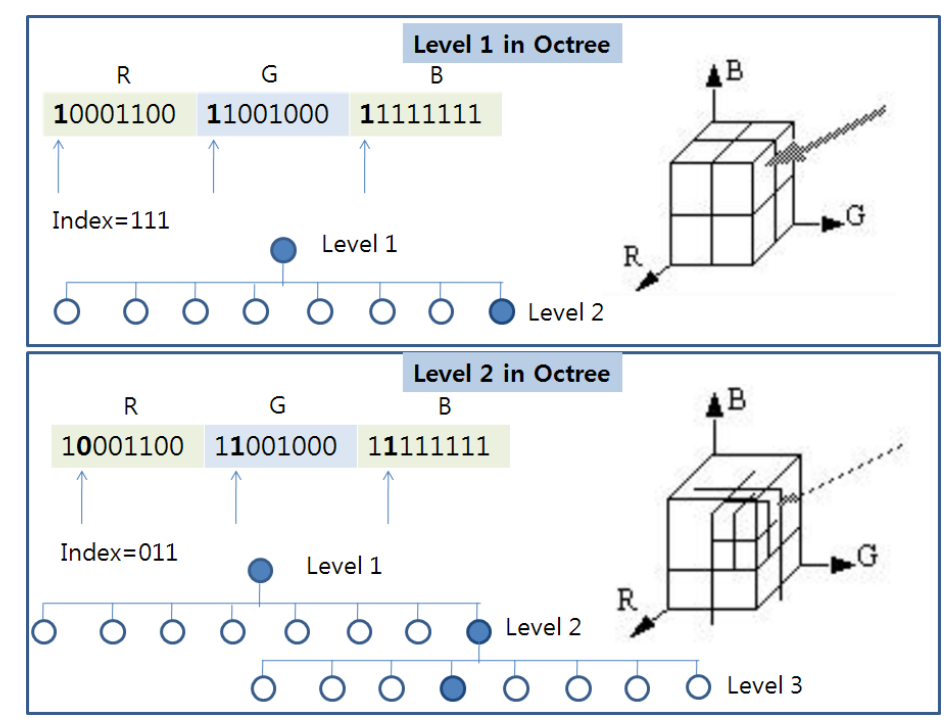

그림 3. RGB 값과 $\mathrm{RGB}$ 큐브 공간 간의 대응 관계

Fig. 3. Mapping an RGB value into the RGB cube/octree 
the RGB cube and octree.

The octree-based quantization is done in three phases:

\section{Step 1. Evaluation of the major colors:}

At the beginning, the octree is empty. Every color that occurs in the image is now inserted by generating a leaf in level eight. Every time the number of leaves exceeds $K$, the octree is reduced. The reduction begins at the bottom of the octree, and is executed by substituting some leaf nodes by their parent node.

\section{Step 2. Filling the color table:}

At the end, the $K$ leaves of the octree contain the colors for the color table. Its own color index of the color table is stored in every leaf node of the octree.

Step 3. Mapping the original colors onto the major colors: Trying to find any original color in the reduced octree will end at a leaf in some depth. This leaf node contains a color very similar to the one in search, and would be its major color. Since the index of the color table is stored there, no further search has to be carried out.

Fig. 4 shows 8 primary colors to be selected from the quantized colors extracted as the result of the octree-based

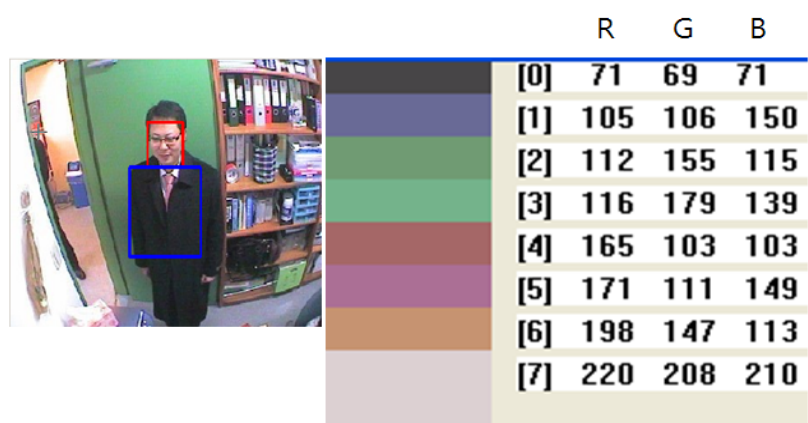

그림 4. 색상 양자화를 통하여 결정된 의상 영역의 대표색

Fig. 4. Primary colors of the clothing area to be decided by applying the color quantization quantization of the black suit with a pink tie.

\section{Measuring Color Similarity Based on Non-Static Histograms}

The histograms based on the extracted major colors are constructed and compared to measure the similarity of different clothing images. We divide the clothing area into six sub-regions, and build the histogram for each sub-region based on the quantized colors. The example of six sub-regions is shown in Fig. 5.

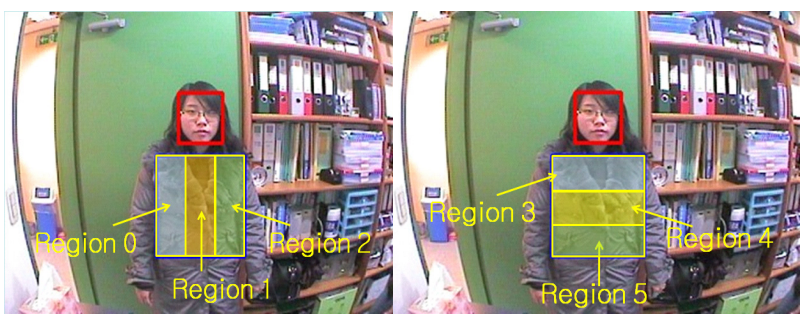

그림 5. 6 개의 세부 의상영역

Fig. 5. Six sub-regions in the clothing area

The histograms for the same sub-region of two images are compared to define the color similarity. If the histograms are derived from a fixed color palette, then two bins at the same index from different histograms still represent the same color. Therefore, the similarity between two different histograms can be easily measured by comparing two matching bins at the same index.

For each image, the color palette generated by octree-based quantization can be unique. Given two clothing images, we may have two different color palettes. As a result, two bins at the same index in different histograms possibly represent two different colors. To measure the similarity between two histograms, we need to find the match bins with similar colors. We also need to adjust the number of bins in the histogram by merging or deleting the bins. Given color palettes $A\left(a_{1}, a_{2}, \ldots, a_{n}\right)$ and $B\left(b_{1}, b_{2}\right.$, 
$\left.\ldots, b_{n}\right)$, where each $a_{i}$ and $b_{i}(1 \leqslant i \leqslant n)$ consists of $(\mathrm{R}, \mathrm{G}$, $\mathrm{B})$ values, the algorithm to measure the similarity is as follows.

Step 1. Generate the color histograms $I$ and $J$ for two clothing images by using given palettes $A$ and $B$, respectively. Let us define the distance between two colors $x$ and $y$ as follows:

$\operatorname{dist}(x, y)=\left(\left(x_{R}-y_{R}\right)^{2}+\left(x_{G}-y_{G}\right)^{2}+\left(x_{B}-y_{B}\right)^{2}\right)^{1 / 2}$

Then, we measure the distance between two histograms $I$ and $J$ with the following equation:

$$
D(I, J)=\operatorname{dist}\left(\frac{1}{n} \sum_{i=1}^{n} a_{i} H_{I, i}, \frac{1}{n} \sum_{i=1}^{n} b_{i} H_{J, i}\right)
$$

The distance $D(I, J)$ is defined as the Euclidean distance between average colors of two histograms. $H_{I, i}$ and $H_{J, i}$ represent the normalized frequencies of the bin $i$ of histogram $I$ and $J$, respectively. $a_{i}$ and $b_{i}$ are colors of the bin $i$ of histograms $I$ and $J$ respectively. If the distance $D(I, J)$ is longer than the given threshold, histograms $I$ and $J$ are determined as totally different pair, and their similarity is decided as the lowest. In this case, 0 is assigned as the similarity value, and after returning the similarity value, algorithm stops in this step. Otherwise, go to step 2 .

Step 2. Determine the match bins of two palettes $A$ and $B$ by computing the shortest Euclidean distance between bin colors. For each bin color in $A, a_{i}$, the closest bin color $b_{j}$ in $B$, which is represented as $\mathrm{M}\left(a_{i}\right)$, is found by the following equation:

$$
j=\operatorname{Argmin}\left(\operatorname{Min}_{1<k<n}\left(\operatorname{dist}\left(a_{i}, b_{k}\right)\right)\right) .
$$

Different bin colors in palette $A$ can have the same closest bin color from $B$.

Step 3. Find many-to-one matches in the result of Step
1 and adjust the palette $A$. If $\mathrm{M}\left(a_{s}\right)=\mathrm{M}\left(a_{t}\right)$, then merge the bins as and at to one bin. When multiple bins are merged into one bin, the frequencies of multiple bins are aggregated together to the merged bin.

Step 4. Find the bin colors of $B$ which is not matched to any bin colors of $A$, and adjust the palette $B$. If the bin color $b$ is not matched, then delete the bin $b$ from palette $B$.

Step 5. After Step 2 and Step 3, we have two adjusted color palettes $A$ and $B$. Now the number of bins in palettes $A$ and $B$ are the same, and matched bins are known. Generate the color histograms $I$ and $J$ for two clothing images by using the adjusted palettes $A$ and $B$, respectively.

Step 6. Measure the color similarity between $I$ and $J$ by using the weighted Euclidean distance. Let $\operatorname{dist}\left(C_{I, i}\right.$, $\left.C_{J, \mathrm{M}(i)}\right)$ represent the Euclidean distance between the color of the bin $i$ of histogram $I$ and the color of the matched bin of histogram $J$. Compute the weighted Euclidean distance between $I$ and $J$ by using Eq. 1 .

$$
S(I, J)=\sum_{i=1}^{m} \operatorname{dist}\left(C_{I, i}, C_{J, M(i)}\right) * \frac{\operatorname{MAX}\left(H_{I, i}, H_{J, M(i)}\right)}{\operatorname{MIN}\left(H_{I, i}, H_{J, M(i)}\right)}
$$

where, $m$ is the number of bins in the histogram. $H_{I, i}$ and $H_{J, \mathrm{M}(i)}$ represent the normalized frequency of the bin $i$ of histogram $I$ and the normalized frequency of the matched bin of histogram $J$, respectively. Since $S(I, J)$ is a distance measure, lower value of $S(I, J)$ means higher similarity. Return $S(I, J)$, and algorithm stops here.

Fig. 6 shows the example of two different histograms with color palettes and match bins. The value that is written in right side of each color box is a component of a 


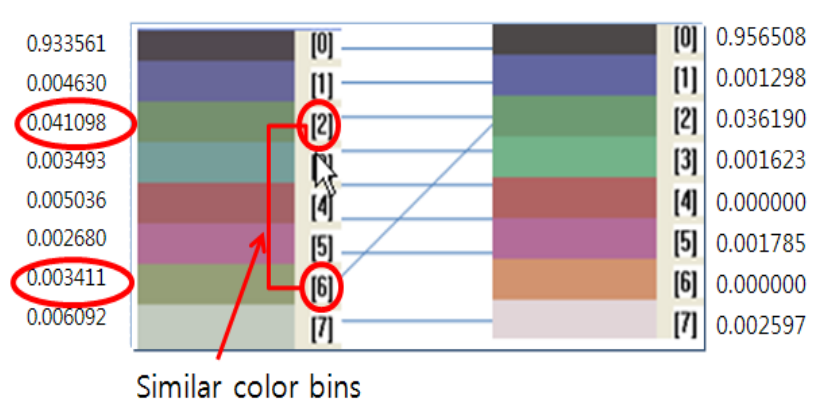

그림 6. 대표색상 팔렛트에 의한 두 히스토그램의 상대도수 및 대응 색상빈 Fig. 6. Two histograms with color palettes and match bins

histogram, and it represents the normalized frequency of corresponding color. The color boxes connected by a line are matched each other. In this example, bin 2 and bin 6 of the left color palette are merged into one. When two bins are merged into one, their corresponding frequencies are added for the merged bin. The bin 6 in the right color palette is deleted.

\section{Experimental Results}

We captured 5 pictures of 42 different clothing by CCD camera at the front door and the elevator, respectively. When the movement of pedestrians is detected, five images are selected from a sequence of images captured by a door CCD camera(Type A). Another five images are selected from the images captured by an elevator CCD camera(Type B). Let us denote the door and elevator images as $D_{s}(i)$ and $E_{s}(i)$, where $i$ is the serial number of the clothing included in the image and $s$ is the serial number of the image $(1 \leqslant s \leqslant 5)$. Given one image for clothing $i$ captured by the door camera as a query image, we retrieve five identical clothing images from $E_{s}(j)$, where $1 \leqslant s \leqslant 5$ and $1 \leqslant j \leqslant 42$, by the following procedure. In the following algorithm, $S\left(Q(i), E_{t}(j)\right)$ is computed by Equation 2 .

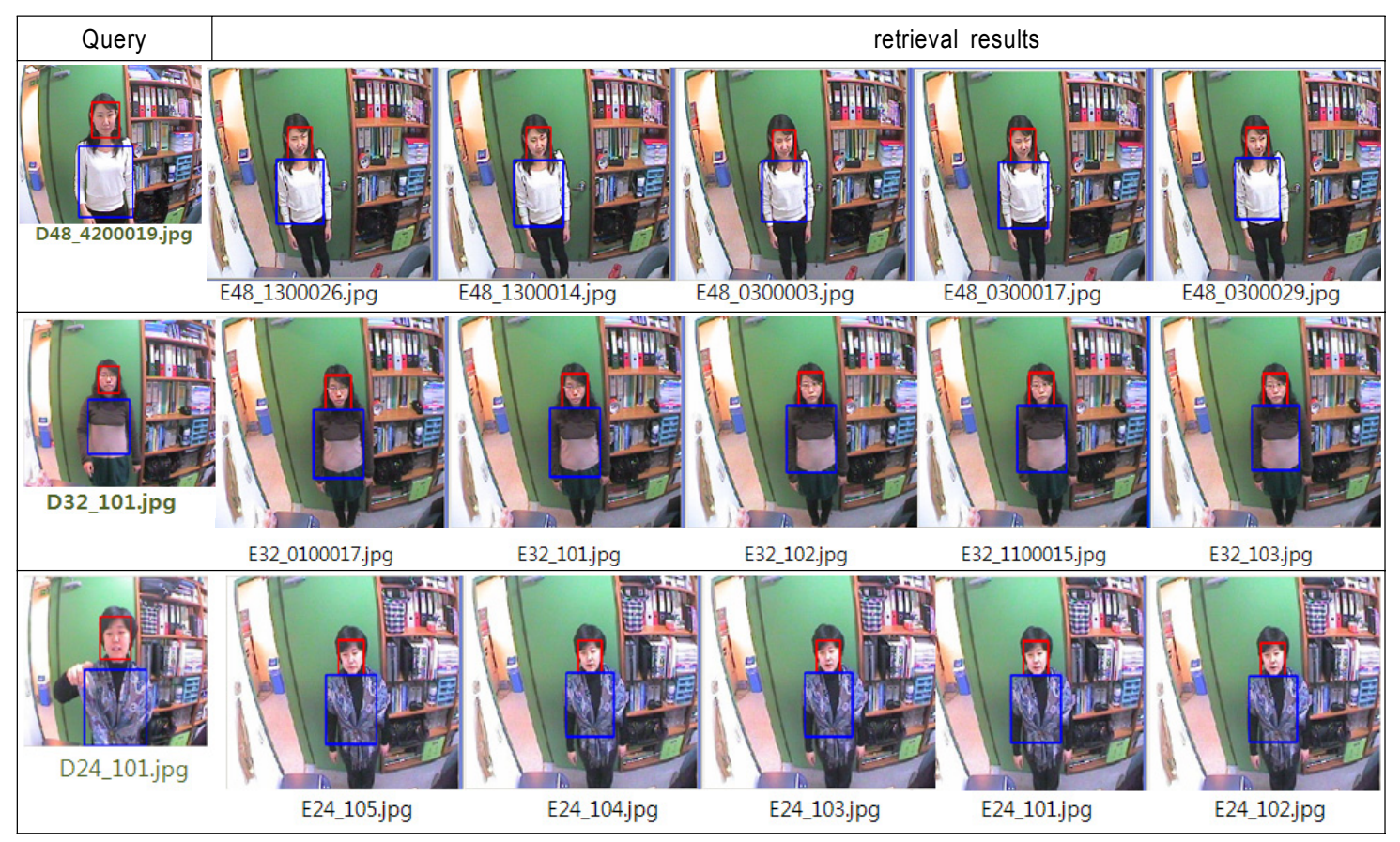

그림 7. 조회 결과가 $100 \%$ 의 정밀도인 사례

Fig. 7. Example of the retrieval with $100 \%$ of precision 


\section{Algorithm: Retrieval of Similar Clothing Images}

Input: $Q(i)$ : A query image selected from door camera images

\section{$E$ : Elevator camera images}

Output: Five similar clothing images

Begin

$$
\begin{gathered}
\text { for } j=1 \text { to } 42 \text { do } \\
\text { for } t=1 \text { to } 5 \text { do } \\
D(j, t)=S\left(Q(i), E_{t}(j)\right)
\end{gathered}
$$

Sort $D(j, t)$ in increasing order and store the result to the array $L_{S}[i]$

Return five elements in the front of $L_{s}[i]$.

\section{End}

Fig. 7 and Fig. 8 show the retrieval result images when we use D1(24), D1(32), D1(48), D1(4) and D1(8) as query images. In the case of D1(24), D1(32) and D1(48), the correct elevator images were retrieved with $100 \%$ of precision. Precision was computed by the following equation:

$$
P=\frac{N_{c}}{N_{c}+N_{f}} \times 100
$$

where, $N_{c}$ is the number of identical clothing images to the query clothing among the five retrieved images and $N_{f}$ $=5-N_{c}$.

In the case of D1(4), the elevator images for D1(1) and D1(5) were retrieved. Clothing D1(4) is the male suit with the pink tie of stripe pattern and elevator images corresponding to D1(1) and D1(5) are also the male suits with the pink tie without a special pattern. When D1(8) of the gray color was a query image, the elevator images $\mathrm{E}(35)$ and $\mathrm{E}(37)$ were retrieved. $\mathrm{E}(35)$ and $\mathrm{E}(37)$ are also gray of the very similar tone to $\mathrm{D} 1(8)$.

In the experiment, our method is compared with two color histogram matching methods: i) HSV cumulative histogram(HSVC)[18] and ii) fixed color index cumulative histogram(FCIC)[19]. HSVC histogram has been widely used because of its robustness against varying illumination. The HSVC histogram method generates one histogram for the whole area, while the FCIC(Fixed color index cumulative) histogram generates multiple local histograms by dividing one clothing area to six local areas. The FCIC method uses eighty fixed colors to define one fixed color palette. In both cases of retrieving the most similar one only and the most

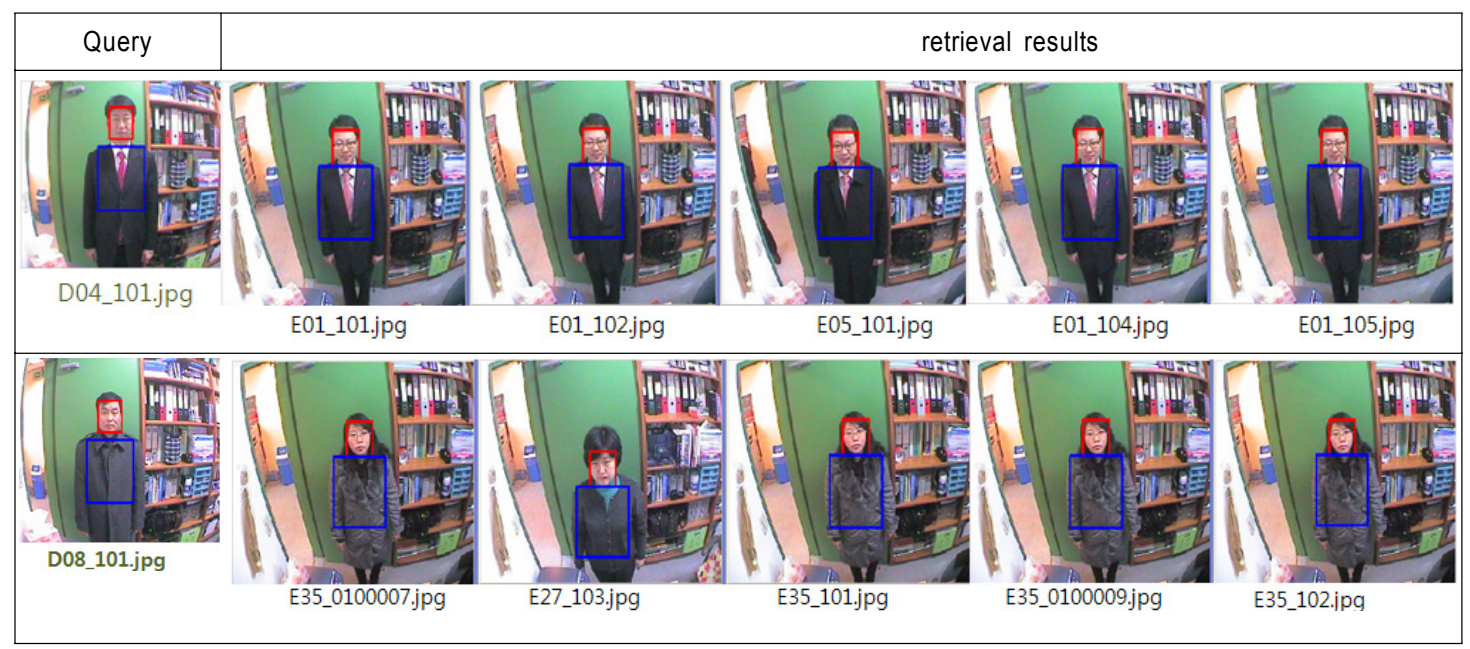

그림 8. 조회 결과가 $0 \%$ 의 정밀도인 사례

Fig. 8. Example of the completely failed retrieval 


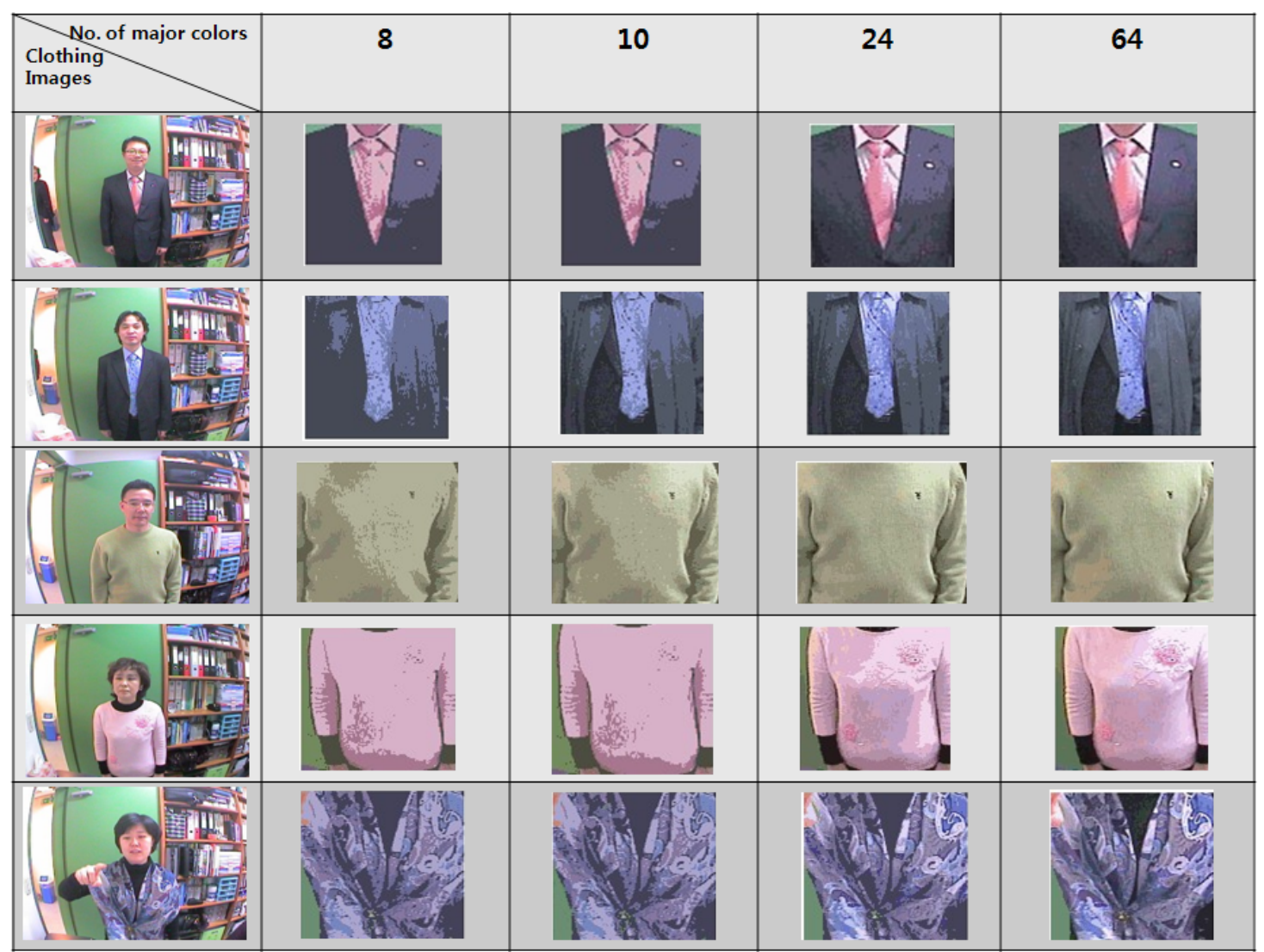

그림 9. 주요 색상수의 차이에 따른 색상 양자화 결과 비교

Fig. 9. Comparison of quantized color images with different numbers of major colors.

similar five images, FCIC showed higher precision than HSVC as shown in Table 1 and Table 2. Compared to these two methods, our method uses the local histograms with non-static color histograms.

According to the maximum number of major colors, we have different results. Experimental results of changing the maximum color parameter of the octree color quantization are shown in Fig. 9.

Table 1 and Table 2 compare the retrieval results according to the different numbers of major colors. When the maximum number of major colors is 64 , the precision of retrieval is the highest. HSVC and FCIC used 85 and 80
표 1. 유사도 1 순위의 동일 의상 조회 인식률 비교

Table 1. Comparison of precision in retrieving one identical clothing

\begin{tabular}{|c|c|c|c|c|c|}
\hline \multirow{2}{*}{$\begin{array}{c}\text { HSVC } \\
\text { Histogram }\end{array}$} & \multirow{2}{*}{$\begin{array}{c}\text { FCIC } \\
\text { Histogram }\end{array}$} & \multicolumn{4}{|c|}{ our method using different number of } \\
(85 bins) & (80 bins) & 8 bins & 10 bins & 24 bins & 64 bins \\
\hline $69.0 \%$ & $76.2 \%$ & $76.2 \%$ & $76.2 \%$ & $76.2 \%$ & $80.6 \%$ \\
\hline
\end{tabular}

표 2. 유사도 5 순위 내의 유사 의상 조회 인식률 비교 Table 2. Comparison of precision in retrieving five identical clothing images

\begin{tabular}{|c|c|c|c|c|c|}
\hline \multirow{2}{*}{$\begin{array}{c}\text { HSVC } \\
\text { Histogram } \\
\text { (85 bins) }\end{array}$} & \multirow{2}{*}{$\begin{array}{c}\text { FCIC } \\
\text { Histogram } \\
\text { (80 bins) }\end{array}$} & \multicolumn{4}{|c|}{$\begin{array}{l}\text { our method using different number of } \\
\text { quantized colors }\end{array}$} \\
\hline & & 8 bins & 10 bins & 24 bins & 64 bins \\
\hline $55.24 \%$ & $57.14 \%$ & $57.62 \%$ & $58.10 \%$ & $59.05 \%$ & $60.48 \%$ \\
\hline
\end{tabular}


color bins, respectively, whereas our method showed the better precision using less than 64 bins. HSVC histogram was able to retreive clothing images of the same chromatic color with higher precision, while it failed to find identical achromatic color with high failure rate. However, our method successively retrieved the clothing images of the same achromatic color as like $\mathrm{D}(48)$ in Fig. 7.

\section{Conclusion}

In this paper, we presented a method to retrieve similar clothing images for given query clothing. To measure the similarity between clothing images, we computed color similarity between two clothing images based on the quantized color histogram. The proposed method extracts major colors from each image using octree-based quantization and composes non-static color palettes. The color palettes are adjusted by finding match bins between two palettes, and then their histograms are compared based on weighted Euclidean distance. Our method provides better precision in retrieval with less number of color bins.

In order to consider the spatial information, we adopted multiple local color histograms for the clothing area. Multiple local histograms were useful to overcome the weakness of the traditional histogram-based approaches that do not consider the spatial information. In order to validate the usefulness of our algorithm, we compared our method with previous approaches through the experiments. Experimental results show that our method provides $13.7 \%$ higher precision compared to the HSV cumulative histogram-based approach.

\section{REFERENCES}

[1] J. Y. Ha, M. Choi, H. Choi, "Content-based Image Retrieval Using Color and Shape", The Journal of the Korean Society of Computer and Information, Vol. 13, No. 1. pp. 117-123, 2008.

[2] K. S. You, H. -H. Yoo, H. S. Kwak, "Histogram Matching
Algorithm for Content-based Image Retrieval", The Journal of the Korea Information and Communication Society, Vol.33. No.1. pp. 45-52, 2008

[3] J. S. Noh, C. H. Baek, K. H. Rhee, "Implementation on the Filters using Color and Intensity for the Content based Image Retrieval", The Journal of the Institute of Electronics Engineers of Korea, Vol.44. No.1. pp. 122-129, 2008.

[4] D. -W. Kim, U. -D. Chang, N. -J. Kwak, Y. -J. Song, "Content-based Image Retrieval Using Object Region with Main Color", The Journal of the Korea Contents Association, Vol.6. No.2. pp.45-49, 2006.

[5] Y. -M. Lee, K. -M. Lee, "A Surveillance System Combining Model-based Multiple Person Tracking and Non-overlapping Camera", The Journal of the Korean Institute of Information Scientists and Engineers, Vol.12. No.4. pp.241-253, 2006.

[6] J. Ryu, Y. Nam, W. -D. Cho, "Tracking People under Occlusion using Multiple Cameras", KCC '08, Vol.35. No.1(c). pp.445-449, 2008.

[7] Q. Iqbal, J. K. Aggarwal, "CIRES:A System for Content-based Retrieval in Digital Image Libraries", International Conference on Control Automation", Robotics and Vision, pp.205-210, 2002.

[8] J. Z. Wang, J. Li, G. Wiederhold, "SIMPLcity: Semantics-Sensitive Integrated Matching for Picture Libraries", IEEE Transactions on Pattern Analysis and Machine Intelligence, Vol. 23. No. 9. pp. 947-963, 2001.

[9] M. J. Swain, D. H. Ballard, "Color Indexing", International Journal of Computer Vision, Vol. 7. No. 1. 1991.

[10] X. Hongli, X. De, G. Yong, "Region-Based Image Retrieval usingColor Coherence Region Vectors", Proceeding of the 7th International Conference on Signal Processing, pp. 761-764, 2004.

[11] K. Kang, Y. Park, Y. Yoon, J. Choi, D. Kim, "Image Retrieval using Spatial Infromation and Color Changing Ratio", Journal of Korea Multimedia Society, Vol. 11. No. 1. pp. 23-33, 2008.

[12] F. Tous, A. Borràs, R. Benavente, R. Baldrich, M. Vanrel, J. Lladós, "Textual Descriptors for Browsing People by Visual Appearance", Lecture Notes in Computer Science, Vol. 2504. pp. 419-429, 2002

[13] D. Kang, J. Ha, "Digital Image Processing using Visual c++", Chapter 11, SitechMedia, 2003.

[14] Y. -J. Choi, K. -J. Kim, W. -D. Cho, "Grid-based Approach for Detecting Head and Hand Regions", ICIC 2007, CCIS 2, pp.1126-1132, 2007.

[15] R. E. Schapier, Y. Singer, "Improved Boosting Algorithms using Confidence-rated Predictions", Machine Learning, Vol. 3, No. 3, pp. 297-336, 1999

[16] M. Gervautz, W. Purgathofer, "A Simple Method for Color Quantization: Octree Quantization", In New Trends in Computer Graphics, pp. 287-293,1990.

[17] M. T. Orchard, C. A. Bouman, "Color Quantization of Images," IEEE Transactions on Signal Processing, Vol. 39. No. 12. pp. 2677-2690, 1991. 
[18] M. Stricker, M. Orengo, "Similarity of Color Images", Storage and Retrieval for Image and Video Databases(SPIE), Vol. 2420, pp. 381-392, 1995.
[19] Y. -J. Choi, K. -J. Kim, Y. Nam, W. -D. Cho, "Retrieval of Identical Clothing Images based on Local Color Histograms", ICCIT 2008, pp. 818-823, 2008.

저 자 소 개

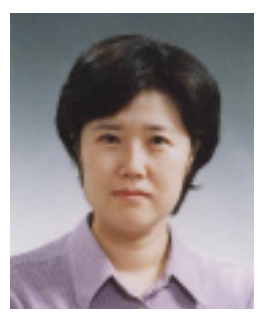

\section{Yoo-Joo Choi}

- 1989년 : Dept. of Computer Science, Ewha Womans University (B.S)

- 1991년 : Dept. of Computer Science, Ewha Womans University (M.S)

- 2005년 : Dept. of Computer Science, Ewha Womans University (Ph.D)

- 1991년 1993년 : Senior Researcher, R\&D Center, KCI Inc.

- 1993년 1999년 : Senior Researcher, R\&D Center, Posdata Inc.

- 2005년 현재 : Associate Professor, Dept. of Computer Science \& Application, Seoul University of Venture \& Information

- 주관심분야 : Image Processing, Medical Imaging, Computer Graphics, HCl, Visual Surveillance System

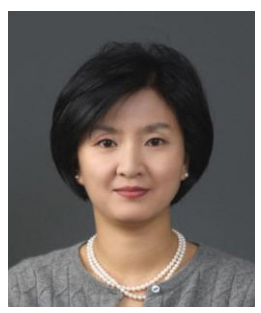

\section{Nam-Mee Moon}

- 1985년 : Dept. of Computer Science, Ewha Womans University (B.S)

- 1987년 : Dept. of Computer Science, Ewha Womans University (M.S)

- 1998년 : Dept. of Computer Science, Ewha Womans University (Ph.D)

- 1998년 : Assistant Professor, Division of Digital Media, Ajou University

- 1999년 2002년 : Research Professor, Manager of Internet Multimedia Research Center, Ewha Womans University

- 2003년 2007년 : Professor, Dept. of Digital Media, Seoul University of Venture \& Information.

- 2008년 현재 : Professor, Dept. of IT Application Technology, Hoseo Graduate School of Venture.

- 주관심분야: Interactive Digital Broadcasting, $\mathrm{HCl}$, e-Learning, Interactive Service

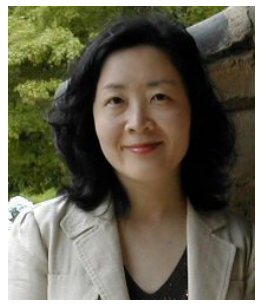

\section{Ku-Jin Kim}

- 1990년 : Dept. of Computer Science, Ewha Womans University (B.S)

- 1992년 : Dept. of Computer Science, KAIST (M.S.)

- 1998년 : Dept. of Computer Science \& Engineering, POSTECH (Ph.D)

- 1998년 2000년 : Dept. of Computer Sciences, Purdue University, USA (PostDoc.)

- 2000년 2002년 : Assistant Professor, Graduate School of Information \& Communication, Ajou University

- 2002년 2003년 : Visiting Assistant Professor, Dept. of Mathematics \& Computer Science, Univ. of Missouri - St. Louis, USA

- 2004년 2008년 : Assistant Professor, Dept. of Computer Engineering, Kyungpook National University

- 2008년 현재 : Associate Professor, Dept. of Computer Engineering, Kyungpook National University

- 주관심분야 : Pattern Recognition, Computer Graphics, Geometric Modeling 